\title{
An Overview of Seminomatous and Non-Seminomatous Germ Cell Testicular Tumors: A Single-center Experience
}

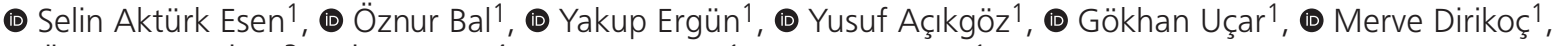

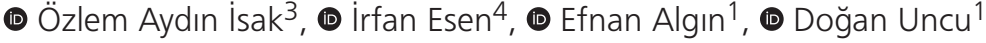 \\ ${ }^{1}$ Ankara City Hospital, Clinic of Medical Oncology, Ankara, Turkey \\ 2Batman Training and Research Hospital, Clinic of Medical Oncology, Batman, Turkey \\ ${ }^{3}$ Ankara Dışkapı Training and Research Hospital, Clinic of Medical Oncology, Ankara, Turkey \\ ${ }^{4}$ Ankara Keçiören Medicalpark Hospital, Clinic of Internal Medicine, Ankara, Turkey
}

\begin{abstract}
Objective: Although germ cell tumors (GCT) are rare malignancies, they are the most common solid tumors in men aged 15-40 years. This study aimed to compare the demographic and clinical characteristics, treatment responses, and survival characteristics of patients with seminomatous GCTs (SGCT) and non-seminomatous GCTs (NSGCTs) followed in our center.

Materials and Methods: Patients with histologically confirmed testicular GCTs and followed up in our hospital between January 2005 and January 2021 were included in this retrospective study. This study was approved by the Institutional Ethics Committee of Ankara City Hospital.

Results: Of the 360 patients, $65.8 \%(n=123)$ had NSGCTs and 34.2\% $(n=237)$ had SGCTs. The median age at diagnosis of the SGCT group was 36 years and that of the NSGCT group was 28 years $(p=0.000)$. Both the diagnostic and postoperative levels of $\beta$-human chorionic gonadotropin were significantly higher in the NSGCT group ( $p=0.000, p=0.000$ respectively). Rates of retroperitoneal lymph node dissection ( $3.3 \%$ vs $9.3 \% ; p=0.036)$, adjuvant radiotherapy (RT) (17.1\% vs $3 \%$; $\mathrm{p}=0.000)$, adjuvant chemotherapy (CT) $(61.1 \%$ vs $40.4 \% ; \mathrm{p}=0.003)$, and metastatic first-line $\mathrm{CT}(22.8 \%$ vs $47.3 \% ; \mathrm{p}=0.000)$ were different between the two groups The 10-year overall survival expectancy rate was $89 \%$ in the SGCT group and $83 \%$ in the NSGCT group.

Conclusion: This study drew attention to the characteristics and treatment responses of patients with NSGCTs and SGCTs. In this study, NSGCTs were diagnosed at an earlier age than SGCTs. The proportion of patients with stage 1 disease at diagnosis was higher in the SGCT group, while that of patients with stage 3 and metastasis at diagnosis were higher in the NSGCT group. In addition, the rates of adjuvant CT and adjuvant RT were higher in the SGCT group, while the metastatic first-line CT rate was higher in the NSGCT group.

Keywords: Testicular germ cell tumors, chemotherapy, radiotherapy, autologous stem-cell transplantation
\end{abstract}

\section{Introduction}

Although germ cell tumors (GCTs) are rare malignancies, they are the most common solid tumors in men aged $15-40$ years (1). Testicular cancers constitute only $0.5 \%$ of all cancers in men. Even if its etiology is not yet fully clarified, risk factors include family history, cryptorchidism, contralateral testicular tumor, infertility, and testicular microlithiasis (2). Moreover, 95\% of testicular cancers are GCTs and $5 \%$ are non-GCTs and various non-specific stromal tumors. Testicular GCTs is divided into two groups, namely, as seminoma and non-seminoma. Nonseminomatous GCTs (NSGCTs) are divided into five subtypes: embryonal carcinomas, yolk sac tumors, choriocarcinomas, teratomas, and mixed GCTs (MGCTs) (3).
The majority of the patients can be treated by orchiectomy and, if necessary, systemic or local treatments (4). Patients with GCTs have excellent survival rates because of advances in chemotherapy (CT), radiotherapy (RT), and surgery (5). A cure is expected in $95 \%$ of all patients with testicular cancer and approximately $80 \%$ of patients with metastatic disease (4).

In this study, we aimed to compare the demographic and clinical characteristics, treatment responses, and survival data of patients with seminomatous GCTs (SGCT) and NSGCTs followed in our center.

\section{Materials and Methods}

This study was approved by the Institutional Ethics Committee of Ankara City Hospital (decision no: El-21-1661). Patients with 
histologically confirmed testicular GCTs and followed up in our hospital between January 2005 and January 2021 were included in this retrospective study. Clinicopathological characteristics of the patients and treatment modalities were recorded from the patient registration database of the hospital. $\beta$-human chorionic gonadotropin (BHCG), lactate dehydrogenase (LDH), and alpha fetoprotein (AFP) levels were measured upon diagnosis and 30 days after orchiectomy. Patients with a second cancer were excluded from the study.

Testicular cancer was staged using the Eighth Tumor, Node, Metastasis staging system developed jointly by the American Joint Committee on Cancer and the Union for International Cancer Control, which applies to both SGCT and NSGCTs (6).

According to the RECIST guidelines, responses were calculated using the following measurements: complete response (CR) (complete resolution of target lesions), partial response (PR) ( $\geq 30 \%$ decrease in the sum of the diameters of the target lesions compared with the baseline), progressive disease (PD) $(\geq 20 \%$ increase in sum of the diameters of the target lesions compared with baseline or new metastatic lesions), and stable disease (SD; neither fitting in PR or PD categories). CR/PR, SD, and PD as per RECIST were independently analyzed (7).

The diagnosis of recurrent testicular GCTs was typically made based on an increase in serum tumor markers or evidence of disease progression on radiographic or physical examinations. Biopsy confirmation was also performed in cases where the recurrence symptom was atypical.

\section{Statistical Analysis}

Statistical analyses were performed using SPSS Statistics version 24.0 (IBM Corp., Armonk, NY, USA). Continuous variables were expressed as median with the $25^{\text {th }}$ percentile and $75^{\text {th }}$ percentile. Categorical variables were presented as percentage. The normality of quantitative data has been analyzed by the Kolmogorov-Smirnov and Shapiro-Wilk tests. The Pearson chisquare test was used for the comparison of categorical variables between two groups, and independent sample t-test or MannWhitney $U$ test was used for comparison of continuous variables between two groups. Survival analysis was calculated according to the Kaplan-Meier (Log rank, Breslow, and Tarone-Ware analyses) method. P-value $<0.05$ was considered significant.

\section{Results}

Of the total 360 patients, $65.8 \%(n=237)$ had NSGCTs and $34.2 \%(n=123)$ had SGCTs. The median age at diagnosis of the SGCT group was 36 years and that of NSGCT group was 28 years $(p=0.000)$. The tumor diameter measure by computed tomography was $4.5 \mathrm{~cm}$ in the SGCT group and $4 \mathrm{~cm}$ in the NSGCT group. No significant difference was found in the mean tumor diameter between the two groups. The median BHCG levels upon diagnosis and after surgery were significantly higher in the NSGCT group ( $p=0.000$ and $p=0.000$, respectively). No difference was noted between the two groups in terms of LDH levels upon diagnosis and after surgery (Table 1).

Of the NSGCTs, $74 \%$ were MGCTs, $12.2 \%$ were embryonal carcinomas, $7.2 \%$ were teratomas, $5.1 \%$ were yolk sac tumors, and $0.8 \%$ was choriocarcinomas. Of the MGCTs, $80.1 \%$ were embryonal carcinomas, $63.5 \%$ were teratomas, $49.7 \%$ were yolk sac tumors, $35.4 \%$ were seminomas, and $11.6 \%$ were choriocarcinomas (Table 2).

The proportion of patients with stage 1 disease at diagnosis was $73.9 \%$ in the SGCT group and $46.8 \%$ in the NSGCT group $(p=0.000)$, while the proportion of patients with stage 3 disease at diagnosis was $9.2 \%$ in the SGCT group and $30.4 \%$ in the NSGCT group $(p=0.000)$. The proportion of patients with stage 2 disease at diagnosis was comparable between the two groups. The proportions of patients with SGCTs and NSGCTs in the good-risk group were $93.4 \%$ and $59.5 \%$, respectively $(p=0.000)$. The proportion of the patients in the intermediate-risk group were $6.4 \%$ and $25.7 \%$, respectively $(p=0.000)$. The rates of patients with positive lymph nodes (LNs) at diagnosis were $27.8 \%$ and $52.3 \%$ in the SGCT and NSGCT groups, respectively. The proportions of patients with metastasis at diagnosis were $0.8 \%$ and $27 \%$ in the SGCT and NSGCT groups, respectively. Moreover, the proportions of patients with LN positivity and metastatic disease were significantly higher in the NSGCT group $(p=0.006$ and $p=0.000$, respectively) (Table 1 ).

The recurrence rate was $17.1 \%$ in the SGCT group and $18.6 \%$ in the NSGCT group. The proportion of patients with recurrence was not different between the two groups $(p=0.727)$. The rate of patients who underwent retroperitoneal LN dissection (RPLND) was significantly higher in the NSGCT group $(9.3 \%, n=22)$ than in the SGCT group $(3.3 \%, n=4)(p=0.036)$. While one patient in the SGCT group had post-CT RPLND and three patients had primary RPLND, 16 patients with NSGCT had post-CT RPLND and six patients had primary RPLND. In patients with SGCT who underwent RPLND, seminoma was detected in one patient (stage 1 disease) and necrosis was detected in three patients (two and one patient has stage 2 and 1 disease, respectively). In patients with NSGCT who underwent RPLND, $27.3 \%$ of the patients had teratomas (four and one patient had stage 2 and 3 disease, respectively), $18.2 \%$ had necrosis (one, two, and one patient had stage 1, 2, and 3 disease, respectively), 31.8\% had other non-seminomatous subtypes (two, three, and two patients had stage 1, 2, and 3 disease, respectively), 9.1\% had seminomas (two patients had stage 3 disease), 9.1\% had reactive LNs (one and one patient had stage 1 and stage 3 disease), and 4.5\% were non-diagnostic (one patient had stage 2 disease) (Table 3). The proportion of patients receiving adjuvant RT was significantly higher in the SGCT group (17.1\%, $n=21)$ than in the NSGCT group $(3 \%, n=7)(p=0.000)$. In the SGCT group, only one patient received testicular RT, while 20 patients received RT for para-aortic \pm iliac $L N s(n=11)$ or inguinal $L N s(n=9)$. All patients with NSGCTs received RT to para-aortic \pm iliac LNs $(n=5)$ or inguinal LNs ( $n=2)$ (Tables 3 and 4).

The proportion of patients receiving adjuvant CT was significantly higher in the SGCT group $(61.1 \%, \mathrm{n}=44)$ than in the NSGCT group $(40.4 \%, n=76)(p=0.003)$. As adjuvant CT in the SGCT group, $31.8 \%$ of the patients received carboplatin and $15.9 \%$ received cisplatin, etoposide, bleomycin (BEP, bleomycin $30 \mathrm{U}$ IV weekly on days 1,8 , and 15 + etoposide $100 \mathrm{mg} / \mathrm{m}^{2} \mathrm{IV}$ on days 1-5 + cisplatin $20 \mathrm{mg} / \mathrm{m}^{2}$ IV on days 1-5/repeat every 21 days) + cisplatin, etoposide (EP, etoposide $100 \mathrm{mg} / \mathrm{m}^{2}$ IV on days 1-5 + cisplatin $20 \mathrm{mg} / \mathrm{m}^{2}$ IV on days 1-5/repeat every 21 days) and 


\begin{tabular}{|c|c|c|c|c|c|c|}
\hline & & \multicolumn{2}{|l|}{ SGCT $n=123(34.2 \%)$} & \multicolumn{2}{|c|}{ NSGCT $n=237(65.8 \%)$} & \multirow[b]{2}{*}{ p-value } \\
\hline & & $\begin{array}{l}\text { Median (minimum; } \\
\text { maximum) }\end{array}$ & $\%$ & $\begin{array}{l}\text { Median (minimum; } \\
\text { maximum) }\end{array}$ & $\%$ & \\
\hline \multicolumn{2}{|l|}{ Follow-up period (years) } & $7.75(0.70 ; 33)$ & & $8.79(0.68 ; 22.08)$ & & \\
\hline \multicolumn{2}{|l|}{ Age at diagnosis (years) } & $36(17 ; 62)$ & & $28(15 ; 67)$ & & 0.000 \\
\hline \multicolumn{2}{|l|}{ Tumor diameter $(\mathrm{cm})$} & $4.50(0.50 ; 12)$ & & $4.00(1 ; 10)$ & & 0.411 \\
\hline \multicolumn{2}{|c|}{ BHCG at diagnosis (IU/mL) } & $2(0.1 ; 2891)$ & & $17(0.1 ; 275486)$ & & 0.000 \\
\hline \multicolumn{2}{|l|}{$\mathrm{LDH}$ at diagnosis (U/L) } & $284(127 ; 4720)$ & & $261(120 ; 5400)$ & & 0.651 \\
\hline \multicolumn{2}{|l|}{ AFP at diagnosis $(\mathrm{ng} / \mathrm{mL})$} & & & $110(1 ; 128000)$ & & \\
\hline \multicolumn{2}{|c|}{ Postoperative BHCG (IU/mL) } & $0.2(0.1 ; 1907)$ & & $2(0.1 ; 596000)$ & & 0.000 \\
\hline \multicolumn{2}{|l|}{ Postoperative LDH (U/L) } & $187(90 ; 1799)$ & & $217(91 ; 3577)$ & & 0.055 \\
\hline \multicolumn{2}{|c|}{ Postoperative AFP $(\mathrm{ng} / \mathrm{mL})$} & & & $10(0.1 ; 116000)$ & & \\
\hline \multirow{2}{*}{ Recurrence } & Yes & & $17.1 \%$ & & $18.6 \%$ & 0.727 \\
\hline & No & & $82.9 \%$ & & $81.4 \%$ & \\
\hline \multirow{3}{*}{ Stage at diagnosis } & Stage 1 & & $73.9 \%$ & & $46.8 \%$ & 0.000 \\
\hline & Stage 2 & & $16.8 \%$ & & $22.8 \%$ & 0.146 \\
\hline & Stage 3 & & $9.2 \%$ & & $30.4 \%$ & 0.000 \\
\hline \multirow{3}{*}{ Risk group } & Good & & $93.4 \%$ & & $59.5 \%$ & 0.000 \\
\hline & Intermediate & & $6.4 \%$ & & $25.7 \%$ & 0.000 \\
\hline & Bad & & & & $14.8 \%$ & \\
\hline \multirow{2}{*}{ Tumor } & T1 & & $61.1 \%$ & & $50.4 \%$ & 0.233 \\
\hline & $\geq \mathrm{T} 2$ & & $38.9 \%$ & & $49.6 \%$ & \\
\hline \multirow{2}{*}{ Node positivity } & Negative & & $72.2 \%$ & & $47.7 \%$ & 0.006 \\
\hline & Positive & & $27.8 \%$ & & $52.3 \%$ & \\
\hline \multirow{2}{*}{ Metastasis at diagnosis } & No & & $99.2 \%$ & & $73.0 \%$ & 0.000 \\
\hline & Yes & & $0.8 \%$ & & $27.0 \%$ & \\
\hline
\end{tabular}

\begin{tabular}{|l|l|l|l|l|}
\hline \multicolumn{5}{|c|}{ Table 2. Subtypes of non-seminomatous germ cell tumors } \\
\hline \multirow{4}{*}{} & MGCTs & $\%$ & & $\%$ \\
\hline & & $74.7 \%$ & Teratoma & $63.5 \%$ \\
\hline \multirow{4}{*}{$\begin{array}{l}\text { Pathological } \\
\text { subtype }\end{array}$} & & $\begin{array}{l}\text { Embryonal } \\
\text { carcinoma }\end{array}$ & $80.1 \%$ \\
\cline { 2 - 5 } & & & Yolk sac tumor & $49.7 \%$ \\
\cline { 2 - 5 } & & & Choriocarcinoma & $11.6 \%$ \\
\cline { 2 - 5 } & Teratoma & $7.2 \%$ & & $35.4 \%$ \\
\cline { 2 - 5 } & Yolk sac tumor & $5.1 \%$ & & \\
\cline { 2 - 5 } & Choriocarcinoma & $0.8 \%$ & & \\
\cline { 2 - 5 } & $\begin{array}{l}\text { Embryonal } \\
\text { carcinoma }\end{array}$ & $12.2 \%$ & & \\
\hline \multirow{4}{*}{ MGCT: Mixed germ cell tumors } & & \multicolumn{2}{|l}{} \\
\hline
\end{tabular}

$52.2 \%$ received BEP. In the NSGCT group, $23.6 \%$ of the patients received $B E P+E P, 73.6 \%$ received $B E P$, and $2.6 \%$ received $E P$. The proportion of patients receiving metastatic first-line CT was significantly higher in the NSGCT group than in the SGCT group ( $47.3 \%$ and $22.8 \%$, respectively) $(p=0.000)$. No difference was found between the two groups in terms of metastatic first-line treatment responses, rates of metastatic second-line treatment responses, metastatic second-line treatment responses, or autologous stem cell transplantation (ASCT) rates. ASCT was performed in four patients in the SGCT group and in 10 patients in the NSGCT group. In the SGCT group, PD was attained in one patient and SD was attained in three patients. In the NSGCT group, two patients had CR, one had PR, three had PD, and four had SD (Table 3).

The 10-year overall survival (OS) expectancy rate was $89 \%$ in the SGCT group and $83 \%$ in the NSGCT group. In the survival analysis, OS did not reach the median in either group (Figure 1).

\section{Discussion}

NSGCTs are seen in men aged 20-30 years, while SGCTs typically occur between age 30-40 years (8). In this study, the median age of the patients diagnosed with SGCTs was 36 years, while those with NSGCTs was 28 years, and a significant difference was found between the two groups. No difference was found in tumor size between the two groups, and $34.2 \%$ of the patients had SGCTs and $65.8 \%$ had NSGCTs. In another study conducted in Turkey, the incidence of NSGCT was higher, reporting $77.6 \%$, similar to our study (9). In a retrospective analysis conducted in 


\begin{tabular}{|c|c|c|c|c|}
\hline \multirow{2}{*}{\multicolumn{2}{|c|}{ n (\%) }} & SGCT & NSGCT & \\
\hline & & n (\%) & $p$-value & \\
\hline \multirow{2}{*}{ RPLND } & Yes & $4(3.3 \%)$ & $22(9.3 \%)$ & 0.036 \\
\hline & No & $119(96.7 \%)$ & 215 (90.7\%) & \\
\hline \multirow{2}{*}{ Adjuvant RT } & Yes & $21(17.1 \%)$ & $7(3 \%)$ & 0.000 \\
\hline & No & $102(82.9 \%)$ & $230(97 \%)$ & \\
\hline Adjuvant CT & & $44(61.1 \%)$ & $76(40.4 \%)$ & 0.003 \\
\hline \multirow{2}{*}{$\begin{array}{l}\text { Metastatic first-line } \\
\text { CT }\end{array}$} & Yes & $28(22.8 \%)$ & 112 (47.3\%) & 0.000 \\
\hline & No & $95(77.2 \%)$ & $125(52.7 \%)$ & \\
\hline \multirow{4}{*}{$\begin{array}{l}\text { Metastatic first-line } \\
\text { CT response }\end{array}$} & $C R$ & $6(21.4 \%)$ & $27(24.1 \%)$ & 0.191 \\
\hline & PR & $13(46.4 \%)$ & $53(47.3 \%)$ & 0.093 \\
\hline & PD & $5(17.9 \%)$ & $17(15.2 \%)$ & 0.586 \\
\hline & SD & $4(14.3 \%)$ & 15 (13.4\%) & 0.503 \\
\hline \multirow{2}{*}{$\begin{array}{l}\text { Metastatic second- } \\
\text { line CT }\end{array}$} & Yes & $20(16.3 \%)$ & $41(17.3 \%)$ & 0.803 \\
\hline & No & $103(83.7 \%)$ & 196 (82.7\%) & \\
\hline \multirow{4}{*}{$\begin{array}{l}\text { Metastatic second- } \\
\text { line CT response }\end{array}$} & $C R$ & $6(30 \%)$ & $7(17.1 \%)$ & 0.247 \\
\hline & PR & $8(40 \%)$ & $16(39 \%)$ & 0.942 \\
\hline & PD & $3(15 \%)$ & $9(22 \%)$ & 0.525 \\
\hline & SD & $3(15 \%)$ & $9(22 \%)$ & 0.525 \\
\hline \multirow{2}{*}{ ASCT } & Yes & $4(3.3 \%)$ & $10(4.2 \%)$ & 0.653 \\
\hline & No & $119(96.7)$ & 227 (95.8\%) & \\
\hline \multirow{4}{*}{ ASCT response } & $\mathrm{CR}$ & $0(0 \%)$ & $2(20 \%)$ & \\
\hline & PR & $0(0 \%)$ & $1(10 \%)$ & \\
\hline & PD & $1(25 \%)$ & $3(30 \%)$ & \\
\hline & SD & $3(75 \%)$ & $4(40 \%)$ & \\
\hline \multicolumn{5}{|c|}{$\begin{array}{l}\text { SGCT: Seminomatous germ cell tumors, NSGCT: Non-seminomatous germ cell } \\
\text { tumors, RPLND: Retroperitoneal lymph node dissection, CT: Chemotherapy, } \\
\text { RT: Radiotherapy, ASCT: Autologous stem cell transplantation, CR: Complete } \\
\text { response, PR: Partial response, PD: Progressive disease, SD: Stable disease }\end{array}$} \\
\hline
\end{tabular}

Table 4. Adjuvant radiotherapy localization and adjuvant chemotherapy regimens

\begin{tabular}{|l|l|l|l|}
\hline \multirow{4}{*}{ Adjuvant RT location } & Para-aortic +iliac LN & $11(52.3 \%)$ & $5(71.3 \%)$ \\
\cline { 2 - 4 } & Testicular & $1(4.76 \%)$ & $0(0 \%)$ \\
\cline { 2 - 4 } & Inguinal LN & $9(42.8 \%)$ & $2(28.7 \%)$ \\
\hline \multirow{4}{*}{ Adjuvant CT } & Carboplatin (AUC 7) & $14(31.8 \%)$ & $0(0 \%)$ \\
\cline { 2 - 4 } & BEP+EP & $7(15.9 \%)$ & $18(23.6 \%)$ \\
\cline { 2 - 4 } & BEP & $23(52.2 \%)$ & $56(73.6 \%)$ \\
\cline { 2 - 4 } & EP & $0(0 \%)$ & $2(2.6 \%)$ \\
\hline
\end{tabular}

BEP: Bleomycin $30 \mathrm{U}$ IV weekly on days 1,8 , and $15+$ etoposide $100 \mathrm{mg} / \mathrm{m}^{2}$ IV on days $1-5+$ cisplatin $20 \mathrm{mg} / \mathrm{m}^{2}$ IV on days $1-5 /$ repeat every 21 days, EP:

Etoposide $100 \mathrm{mg} / \mathrm{m}^{2} \mathrm{IV}$ on days $1-5+$ cisplatin $20 \mathrm{mg} / \mathrm{m}^{2} \mathrm{IV}$ on days $1-5 /$ repeat every 21 days, AUC: Area under the curve, LN: Lymph node

Germany, SGCTs constituted $64.5 \%$ of all testicular GCTs, while NSGCTs constituted $35.5 \%$ (10). In another study conducted in Japan, seminomas and non-seminomas were found in $46.7 \%$ and $53.3 \%$ of the patients, respectively (11). In another study conducted in Turkey (12), $46.4 \%$ of the patients with testicular tumors had seminomas and $53.6 \%$ had non-seminomas.

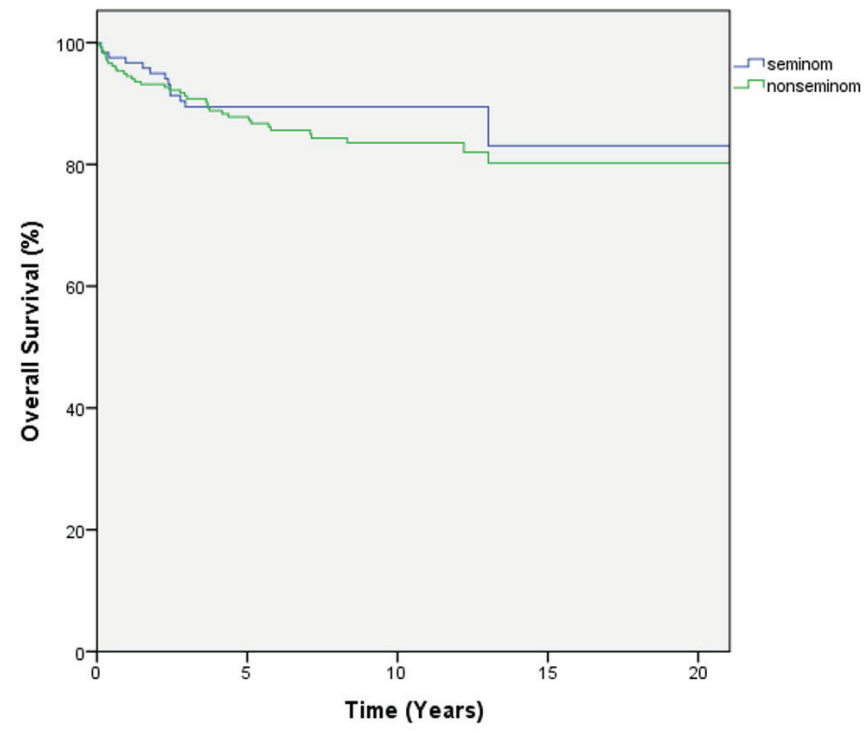

Figure 1. Overall survival curve of seminomatous and non-seminomatous germ cell tumors

In another study (13), these rates were $49.2 \%$ and $50.7 \%$, respectively. Ethnic differences and environmental factors are possible reasons for the differences in the rates of testicular GCTs in different countries.

In the SGCT group, the rates of stage 1, 2, and 3 diseases were $73.9 \%, 16.8 \%$, and $9.2 \%$, respectively. In the NSGCT group, the corresponding rates were $46.8 \%, 22.8 \%$, and $30.4 \%$, respectively. The widespread use of ultrasonography and increased awareness of the public about testicular cancer and testicular self-examination are possible reasons for the higher rates of early-stage testicular tumors compared with metastatic tumors. In addition, testicular cancer may be more easily noticed as it manifests itself with painless scrotal swelling. The rates of stage 1 disease in the SGCT group and stage 3 diseases in the NSGCT group were significantly higher than in those of other stages. In another study, while the rates of stage 1,2 , and 3 diseases were $80.2 \%, 13.5 \%$, and $6.3 \%$, respectively, in the SGCT group, those in the NSGCT group were $53.3 \%$, $27.1 \%$, and $19.6 \%$, respectively (1). Moreover, the proportion of patients with SGCT in the good-risk group was higher than that of patients in the NSGCT group. Patients with moderate risk were higher in the NSGCT group than in the SGCT group. In the present study, $74.7 \%$ of the NSCCTs were MGCT, $12.2 \%$ were embryonal carcinomas, $7.2 \%$ were teratomas, $5.1 \%$ were yolk sac tumor, and $0.8 \%$ was choriocarcinomas. The subcomponents of MGCTs were as follows: $80.1 \%$ were embryonal carcinomas, $63.5 \%$ were teratomas, $49.7 \%$ were yolk sac tumors, $35.4 \%$ were seminomas, and $11.6 \%$ were choriocarcinomas. In a study conducted in Turkey, $77.6 \%$ of the patients had MGCT histology and $82.2 \%$ of them contained histological components of embryonal carcinoma, $53.3 \%$ of teratomas, $49.6 \%$ of yolk sac tumors, $37.8 \%$ of seminomas, and $5.9 \%$ of choriocarcinoma (9). In another study, approximately $65.3 \%$ of NSGCTs were MGCT, $18.7 \%$ were embryonal carcinomas, $7.2 \%$ were yolk sac tumors, $4.5 \%$ were teratomas, and $1.6 \%$ was choriocarcinomas (13). 
In the present study, BHCG levels measured at diagnosis and in the postoperative period were significantly higher in the NSGCT group than in the SGCT group, but no significant difference was found between the two groups in terms of LDH levels. In similar study, while HCG levels were higher in the non-seminoma group at diagnosis, no difference was found between the seminoma and non-seminoma groups in terms of LDH (1).

The American Urological Association (AUA) guidelines on testicular cancer and European Association of Urology (EAU) guidelines in testicular cancer recommend active surveillance after orchiectomy in stage 1 SGCTs and stage 1 NSGCTs $(14,15)$. Adjuvant CT may be a good option in high-risk cases to reduce the risk of recurrence (16). If adjuvant CT will be given, one course of carboplatin (area under curve 7) CT for SGCT and one course of BEP CT for NSGCT can be considered $(14,15)$. In this young patient group, in addition to recurrence, long-term side effects of the treatments, such as cardiovascular events and secondary malignancy, should not be ignored (17). The ratio of patients receiving adjuvant $\mathrm{CT}$ was higher in the SGCT group, while proportion of patients receiving metastatic firstline CT was higher in the NSGCT group. Metastatic second-line $\mathrm{CT}$ rates were comparable between the two groups. However, CT response rates in patients receiving first- and second-line CT were not different between the two groups. In this study, the recurrence rates after adjuvant therapy were comparable in both groups. In addition, $81 \%$ and $88.6 \%$ of the patients diagnosed with recurrent SGCTs and NSGCT, respectively, had relapsed within 3 years. Kollmannsberger et al. (18) reported that the recurrence rate was the highest in the first 3 years after adjuvant $\mathrm{CT}$ in these patients.

Post-CT resection of residual masses or RPLND is often associated with normalization of tumor markers and long-term survival (19). The EAU guideline recommends nerve-sparing RPLND to highly selected patients with stage 1B NSGCTs, i.e., those with contraindication to adjuvant $\mathrm{CT}$ and unwilling to accept surveillance (strong recommendation), and primary RPLND in men with post-pubertal teratomas with somatic malignant components (weak recommendation) (15).

According to the AUA guideline recommendation for patients with stage 1A NSGCTs, RPLND or one cycle of BEP CT is an effective and appropriate alternative treatment option for patients who does not accept surveillance or had incompatible status (14). The guideline offers surveillance, RPLND or one or two cycles of BEP CT based on shared decision-making for patients with stage 1B NSGCTs (14). The AUA guideline also stated that clinicians may offer RPLND as an alternative to CT in select patients with clinical stage 2B NSGCTs with normal post-orchiectomy serum AFP and beta-hCG. To date, little data are available on outcomes for men receiving RPLND as primary treatment for SGCTs (14). In a study using data from the Surveillance, Epidemiology, and Final Results program, the rates of RPLND were $1.3 \%$ in stage 1 disease and $10.6 \%$ in stage 2 disease in men diagnosed with testicular SGCTs between 1988 and 2013 in the United States (20). In this study, RPLND was performed in $3.3 \%$ of the patients with SGCTs and 9.3 of those with NSGCTs. In all patients who underwent RPLND, the viable tumor, necrosis, and reactive $\mathrm{LN}$ and non-diagnostic $\mathrm{LN}$ rates were $57.6 \%, 30.7 \%$, and $11.5 \%$, respectively. In a single-center analysis of 504 patients with NSGCTs who underwent RPLND, $51 \%$ had fibrosis/necrosis, $37 \%$ had teratomas, and $15 \%$ had viable GCTs (21). Similar results were reported in another study (22). Seminomatous GCTs are extremely sensitive to RT, while NSGCTs are more radioresistant. In the present study, $17.1 \%$ of the patients with SGCTs and $3 \%$ with NSGCTs received adjuvant RT. In the SGCT group, only one patient received testicular RT, while 20 patients received RT for para-aortic \pm iliac LNs. All patients with NSGCTs received RT to para-aortic \pm iliac LNs.

In this study, a total of 14 patients had undergone ASCT. While 3 of 14 patients received high-dose CT + ASCT as third-line therapy, 11 patients received second-line therapy. Moreover, $2 \%$ of the 14 patients had CR, 1 had PR, 7 had SD, and 4 had PD. Randomized studies have reported no improvement in high-dose CT outcomes with ASCT $(23,24)$. However, in nonrandomized studies, better results have been reported when high-dose CT/ASCT was used as second-line CT, rather than third-line CT. In other studies, treatment-related mortality was $<5 \%$, and long-term disease-free survival was between $40 \%$ and $70 \%(24,25,26)$.

In the present study, the 10-year OS expectancy rate was $89 \%$ in the SGCT group and $83 \%$ in the NSGCT group. The median OS could not be reached. Although the incidence of testicular cancer has increased, related mortality decreased over time (27). In the 1970s, the application of cisplatin-based CTs decreased the mortality rate while increasing the life expectancy rate to $95 \%$ (28). In one study, the 10-year OS expectancy rate was $90.8 \%$ in patients with testicular cancer diagnosed at age $<50$ years, while the OS expectancy rate was $80.4 \%$ in those diagnosed at age $>50$ years. In another study, the 5-year OS expectancy rates were $93.8 \%$ and $87.9 \%$ in the SGCT and NSGCT groups, respectively (29).

\section{Study Limitations}

The retrospective study design was the most important limitation of this study. Thus, surgical techniques, RT doses, and number of CT cycles could not be found in patient records.

\section{Conclusion}

In this study, clinical and laboratory characteristics, treatment responses, and survival characteristics of patients with SGCTs and NSGCTs who were followed up in our center were examined. The aim was to draw attention to the similar and different characteristics and treatment responses between the two groups. Compared with SGCTs, NSGCTs were diagnosed at an earlier age. The proportion of patients with stage 1 disease at diagnosis was higher in the SGCT group, and those with stage 3 disease and metastasis at diagnosis were higher in the NSGCT group. In addition, the rates of adjuvant CT and adjuvant RT were higher in the SGCT group, while RPLND and metastatic first-line CT rates were higher in the NSGCT group. Evaluation of the clinical laboratory and survival data of 360 patients with GCTs was the strength of the study. However, studies involving a large patient population from different ethnic and geographical regions are warranted. 


\section{Acknowledgements}

Publication: The results of the study were not published in full or in part in form of abstracts.

Contribution: There is not any contributors who may not be listed as authors.

Conflict of Interest: No conflict of interest was declared by the authors.

Financial Disclosure: The authors declared that this study received no financial support.

\section{Ethics}

Ethics Committee Approval: This study was approved by the Institutional Ethics Committee of Ankara City Hospital (decision no: El-21-1661).

Informed Consent: Retrospective study.

Peer-review: Externally and internally peer-reviewed.

\section{Authorship Contributions}

Critical Review: Y.E., Y.A., G.U., E.A., Concept: Ö.B., Design: S.A.E., Ö.B., M.D., D.U., Data Collection or Processing: S.A.E., Ö.B., M.D., Ö.A.I., Analysis or Interpretation: S.A.E., Y.E., Y.A., I.E., Literature Search: S.A.E., G.U., Writing: S.A.E., İ.E.

\section{References}

1. Rothermundt C, Thurneysen C, Cathomas R, et al. Baseline characteristics and patterns of care in testicular cancer patients: first data from the Swiss Austrian German Testicular Cancer Cohort Study (SAG TCCS). Swiss Med Wkly 2018;148:w14640.

2. Leblanc L, Lagrange F, Lecoanet $P$, et al. Testicular microlithiasis and testicular tumor: a review of the literature. Basic Clin Androl 2018;28:8.

3. Vaz RM, Bordenali G, Bibancos M. Testicular cancer-surgical treatment. Front Endocrinol (Lausanne) 2019;10:308.

4. Hanna NH, Einhorn LH. Testicular cancer--discoveries and updates. N Engl J Med 2014;371:2005-2016.

5. Schwen ZR, Gupta M, Pierorazio PM. A review of outcomes and technique for the robotic-assisted laparoscopic retroperitoneal lymph node dissection for testicular cancer. Adv Urol 2018;2018:2146080.

6. Brimo F, Srigley JR, Ryan CJ, et al. Testis. In: Amin MB, ed. AJCC Cancer Staging Manual. 8th ed. New York Springer; 2017. p.727.

7. Eisenhauer EA, Therasse P, Bogaerts J, et al. New response evaluation criteria in solid tumours: revised RECIST guideline (version 1.1). Eur J Cancer 2009; 45: 228-247. 2008/12/23.

8. Sarici $\mathrm{H}$, Telli $\mathrm{O}$, Eroglu M. Bilateral testicular germ cell tumors. Turk J Urol 2013;39:249-252. 2013/12/01.

9. Erol Gülseven M, Üyetürk Ü, Geredeli Ç. Evaluation of General Features of Patients with Testicular Cancer. Bull Urooncol 2020;19:141-145.

10. Brandt MP, Gust KM, Bon D, et al. Trend analysis and regional tumor incidence in Germany for testicular cancer between 2003 and 2014. Andrology 2019;7:408-414. 2019/07/17.

11. Yamashita S, Koyama J, Goto T, et al. Trends in age and histology of testicular cancer from 1980-2019: a single-center Study. Tohoku J Exp Med 2020;252:219-224. 2020/11/06.
12. Karaçetin D, Maral Ö, Ökten B, Yalçin B, Incekara O. Prognostic factors and treatment results in testicular cancer. SEH Tıp Bülteni 2008;42:22-26.

13. Gürsoy P, Çakar B, Gökmen E, et al. Epidemiological and overall survival characteristics of testicular cancers in Ege University Hospital database. Ege Tıp Dergisi 2019;58:126-132.

14. Stephenson A, Eggener SE, Bass EB, et al. Diagnosis and treatment of early stage testicular cancer: AUA guideline. J Urol 2019;202:272-281.

15. European Association of Urology (EAU) testicular cancer 2019. Available from: https://uroweb.org/guideline/testicular-cancer/

16. Mortensen MS, Bandak M, Kier MG, et al. Surveillance versus adjuvant radiotherapy for patients with high-risk stage I seminoma. Cancer 2017; 123:1212-1218.

17. Travis LB, Beard C, Allan JM, et al. Testicular cancer survivorship: research strategies and recommendations. I Natl Cancer Inst 2010;102:1114-1130.

18. Kollmannsberger $C$, Tandstad T, Bedard PL, et al. Patterns of relapse in patients with clinical stage I testicular cancer managed with active surveillance. J Clin Oncol 2015;33:51-57.

19. Fizazi K, Oldenburg J, Dunant A, et al. Assessing prognosis and optimizing treatment in patients with postchemotherapy viable nonseminomatous germ-cell tumors (NSGCT): results of the sCR2 international study. Ann Oncol 2008;19:259-264.

20. Patel HD, Joice GA, Schwen ZR, et al. Retroperitoneal lymph node dissection for testicular seminomas: population-based practice and survival outcomes. World J Urol 2018;36:73-78.

21. Carver BS, Serio AM, Bajorin D, et al. Improved clinical outcome in recent years for men with metastatic nonseminomatous germ cell tumors. J Clin Oncol 2007;25:5603-5608.

22. Heidenreich A, Pfister D, Witthuhn R, et al. Postchemotherapy retroperitoneal lymph node dissection in advanced testicular cancer: radical or modified template resection. Eur Urol 2009;55:217-224.

23. Lorch A, Kleinhans A, Kramar A, et al. Sequential versus single highdose chemotherapy in patients with relapsed or refractory germ cell tumors: long-term results of a prospective randomized trial. J Clin Oncol 2012;30:800-805.

24. Lorch A, Bascoul-Mollevi C, Kramar A, et al. Conventional-dose versus high-dose chemotherapy as first salvage treatment in male patients with metastatic germ cell tumors: evidence from a large international database. J Clin Oncol 2011;29:2178-2184.

25. Bhatia S, Abonour R, Porcu P, et al. High-dose chemotherapy as initial salvage chemotherapy in patients with relapsed testicular cancer. I Clin Oncol 2000;18:3346-3351.

26. Beyer J, Stenning S, Gerl A, et al. High-dose versus conventionaldose chemotherapy as first-salvage treatment in patients with nonseminomatous germ-cell tumors: a matched-pair analysis. Ann Oncol 2002;13:599-605.

27. Stang A, Rusner C, Eisinger B, et al. Subtype-specific incidence of testicular cancer in Germany: a pooled analysis of nine populationbased cancer registries. Int J Androl 2009;32:306-316.

28. Bosetti C, Bertuccio P, Chatenoud L, et al. Trends in mortality from urologic cancers in Europe, 1970-2008. Eur Urol 2011;60:1-15.

29. Drevinskaite $M$, Patasius $A$, Kincius $M$, et al. A population-based analysis of incidence, mortality, and survival in testicular cancer patients in Lithuania. Medicina (Kaunas) 2019;55:552. 